\title{
Analyse eines bindemittelhaltigen Pumpversatzes
}

\author{
Maximilian Schall und Anja Moser
}

Lehrstuhl für Bergbaukunde, Bergtechnik und Bergwirtschaft, Montanuniversität Leoben, Leoben, Österreich

Eingegangen 28. April 2017; angenommen 2. Mai 2017; online publiziert 15. Mai 2017

\begin{abstract}
Zusammenfassung: Im Zuge einer Bakkalaureatsarbeit am Lehrstuhl für Bergbaukunde, Bergtechnik und Bergwirtschaft der Montanuniversität Leoben wurde ein bindemittelhaltiger Pumpversatz auf seine Festigkeitsentwicklung in den ersten 90 Tagen nach der Versatzherstellung analysiert. Dabei wurde anhand verschiedener Probengeometrien und -größen versucht, ein optimiertes Prüfverfahren für den betroffenen Bergbau zu entwickeln und den Einfluss der Bindemittelmenge und -art auf die einaxiale Druckfestigkeit zu ermitteln. In der vorliegenden Arbeit werden die wesentlichen Erkenntnisse dieser Bakkalaureatsarbeit dargestellt. Hierzu werden in den folgenden Kapiteln die Erstellung der Proben und deren Prüfung beschrieben. Einen wesentlichen Punkt dieser Arbeit stellt die abschließende Analyse dar, in welcher die erzielten Ergebnisse aus den Druckfestigkeitsprüfungen interpretiert werden. Daraus können Schlüsse für das betroffene Bergwerk gezogen werden.
\end{abstract}

Schlüsselwörter: Versatz, Untertagebergbau, Einaxiale Druckfestigkeit

\section{Analysis of a Paste Fill Mixture}

Abstract: In the framework of a bachelor thesis at the Chair of Mining Engineering and Mineral Economics of the Montanuniversität Leoben a paste fill mixture was analysed concerning its strength development over 90 days after backfill preparation. In this context, different sample geometries and sizes were used in order to obtain an optimum backfill testing procedure for the concerned underground mine. Further on the influence of the amount and type of binding agent on the uniaxial compressive strength was investigated. The present paper presents the main findings of the bachelor thesis. For this purpose, the preparation and testing of the samples are described. An essential part of this

\section{Schall ( $\square)$}

Lehrstuhl für Bergbaukunde, Bergtechnik und Bergwirtschaft,

Montanuniversität Leoben,

Franz Josef-Straße 18,

8700 Leoben, Österreich

maximilian-johannes.schall@stud.unileoben.ac.at study is the final analysis, where the achieved results from the uniaxial compression tests are discussed and conclusions for the underground mine concerned are drawn.

Keywords: Backfill, Underground mining, Uniaxial compressive strength

\section{Einleitung}

Das Verfüllen bzw. Versetzen von untertägigen Hohlräumen im Bergbau stellt mittlerweile einen wesentlichen Bestandteil der betrieblichen Aktivitäten in untertägigen Bergbaubetrieben dar. Unter "Versetzen“ bzw. "Versatz" versteht man alle Tätigkeiten, die mit dem teilweisen oder vollständigen Verfüllen von durch Bergbau geschaffenen Hohlraum untertage verbunden sind [1].

Dabei versteht man unter Versatzmaterial alle Stoffe, die man zur Herstellung des Versatzproduktes verwendet, welches in weiterer Folge in den Hohlraum eingebracht wird und dort den Versatzkörper bildet. Zum Versatzkörper zählen auch Lagerstätten- und Nebengesteinsbestandteile im Hohlraum, welche mit dem Versatz je nach Versatzart einen zusammenhängenden Körper bilden [1]. Das Versatzprodukt setzt sich aus einem Zuschlagsstoff (beispielsweise beim Abbau anfallendes Taubmaterial, Aufbereitungsabgänge, Schotter, Sand ...) und je nach Versatzart aus Wasser bzw. Wasser und Bindemittel (Pumpversatz, Spülversatz) zusammen [2].

In vielen Fällen ist der Haupteinsatzzweck des Versatzes die Stabilisierung des Grubengebäudes, wobei es vielfältige Einsatzgründe für Versatz gibt [3]. Aufgrund der Zusammensetzung des Versatzproduktes (häufig Taubmaterial oder Aufbereitungsabgänge) stellt die Gewährleistung von konstanten Versatzeigenschaften eine große Herausforderung dar. Diese sind jedoch von Nöten, da die Auslegung von Versatztransportsystem und Abbautätigkeiten auf Basis von definierten Versatzeigenschaften erfolgt und Änderungen in der Versatzbeschaffenheit und seinen mechanischen Eigenschaften negative Folgen für Arbeitssicherheit und Produktivität im untertägigen Bergbau haben können. 
Aus diesem Grund muss durch ein geeignetes Probenahme- und Prüfsystem die regelmäßige Kontrolle der Versatzeigenschaften erfolgen.

So wie das Versatzmaterial bzw. das gesamte Versatzsystem eines Bergwerkes den dort herrschenden Bedingungen angepasst werden muss, müssen auch die Versatzbeprobung und -prüfung auf den jeweiligen Betrieb abgestimmt werden. Als Vergleichs- oder Referenzwert für die mechanischen Eigenschaften von Versatz wird zu diesem Zweck häufig die einaxiale Druckfestigkeit herangezogen. Im Zuge der gegenständlichen Bakkalaureatsarbeit wurde ein bindemittelhaltiger Pumpversatz auf die Entwicklung der einaxialen Druckfestigkeit (Uniaxial Compressive Strength - UCS) in den ersten 90 Tagen analysiert. Das Ziel dieser Arbeit war es, durch einen Versuchsaufbau mit 3 verschieden Versatzmischungen herauszufinden, wie sich die Festigkeit verändert, wenn man den Bindemittelanteil im Versatz variiert. Zusätzlich sollte im Zuge dieser Arbeit festgestellt werden, welche Probengröße für die Prüfung der UCS des verwendeten Versatzstoffes ausreichend genaue Ergebnisse erreicht und wie die Proben zu diesem Zwecke hergestellt werden sollen.

\section{Prüfung von Versatzeigenschaften}

Da die Hauptzuschlagstoffe für Versatzprodukte Taubmaterial bzw. Aufbereitungsabgänge sind, welche in den meisten Fällen keine konstanten Eigenschaften aufweisen, stellt die Sicherstellung von definierten Versatzeigenschaften eine große Herausforderung dar. Dies wirkt sich in weiterer Folge auch auf die Auswahl von adäquaten Prüfmethoden für die Feststellung der geforderten Versatzeigenschaften aus. Meist werden Prüfmethoden für Versatz an Regelwerke und Normen der Betontechnik, für Boden oder Gestein angelehnt, da es aufgrund der unterschiedlichen Versatzmaterialien und -einsatzbedingungen nach wie vor kein Regelwerk für Versatzmaterial gibt [4]. Hier muss auch erwähnt werden, dass Ergebnisse aus Laborversuchen nur bedingt zur Beschreibung des In-situVerhaltens herangezogen werden können, da es sich um Tests zur Feststellung der Versatzprodukteigenschaften und nicht um Tests zur Feststellung des Einflusses der Eigenschaften auf die Versatzwirkung handelt. Laborversuche werden unter Normbedingungen bezüglich Lagerung und Versuchsablauf durchgeführt, was nicht den Bedingungen im untertägigen Bergbau entspricht. Die Ergebnisse können herangezogen werden, um Versatzeigenschaften festzustellen und erlauben Rückschlüsse auf zu erwartendes In-situ-Verhalten, können jedoch nicht 1:1 auf dieselben umgelegt werden. Wichtig ist hier das Bewusstsein über die unterschiedlichen Bedingungen und eine sorgfältige Analyse und Interpretation der Ergebnisse auf Basis der Insitu-Bedingungen [3].

\section{Zusammensetzung der Mischungen}

Beim untersuchten bindemittelhaltigen Pumpversatz handelt es sich um eine Mischung aus Zement, AHWZ (auf- bereiteter, hydraulisch wirksamer Betonzusatzstoff), Zuschlagstoffen und Wasser.

Bei den drei im Rahmen dieser Arbeit getesteten Mischungen wurde lediglich der Bindemittelanteil variiert. Dabei wurde eine Mischung hergestellt, die der Standardrezeptur des Betriebes entspricht ("Mischung 1“). Die anderen zwei Mischungen ("Mischung 2" und "Mischung 3") enthielten weniger Zement und mehr AHWZ als die Standardmischung (Zementanteil Mischung $1>$ Zementanteil Mischung 2 > Zementanteil Mischung 3). Das hat zur Folge, dass alle drei Mischungen die gleiche Gesamtmasse besitzen, jedoch unterschiedliche Bindemittelgehalte aufweisen. Das liegt daran, dass "AHWZ" nicht zu $100 \%$ als Bindemittel angerechnet werden kann, sondern nach der k-WertMethode mit einem gewissen Faktor multipliziert werden muss (meist 0,8), um den anrechenbaren Bindemittelwert zu erhalten (ÖNORM EN 12390-1). Obwohl sich aufgrund der Variation des Zement- und AHWZ-Wertes nur geringe Unterschiede in den anrechenbaren Bindemittelmengen ergaben, stellten sich in der Festigkeitsentwicklung der ersten 90 Tage signifikante Änderungen heraus, wie in den folgenden Kapiteln beschrieben wird.

\section{Probengeometrie, Herstellung und Lagerung}

Die einaxiale Druckfestigkeitsprüfung des Versatzes im betreffenden Betrieb erfolgte ursprünglich mittels Zylinderproben mit einem Durchmesser (D) von $100 \mathrm{~mm}$ und einer Höhe (H) von $200 \mathrm{~mm}$. Dabei wurde bei der Herstellung der Proben ein Kunstoffrohr mit Versatz direkt aus der Mischanlage befüllt. Vor der Prüfung wurden 3 bis 4 Proben aus einem Rohr gefertigt. Dabei sind Festigkeitsschwankungen von Proben aus einem Rohr von bis zu $20 \%$ aufgetreten, welche sich zum Teil auf die starke Setzung der Versatzmaterialien im Rohr zurückverfolgen lassen.

Im Falle von Beton schreibt die ÖNORM EN 123901, die oft bei Versatzüberprüfungen herangezogen wird, einen Durchmesser der Zylinderprobe von mindestens 3-mal dem Größtkorn vor. Die ISRM Komission 1979 („International Society for Rock Mechanics Commission on Standardization of Laboratory and Field Tests“) empfiehlt für Gesteinsprüfungen sogar einen Mindestdurchmesser bei Zylinderproben vom 10-fachen des größten Korns [5].

So wurden aufgrund der Vorgaben aus der Betonnorm in dieser Arbeit Prüfkörper mit den Maßen $(\mathrm{D} \times \mathrm{H}$ ) von $115 \times 230 \mathrm{~mm}$ aus Kunststoffrohren hergestellt. Für alle Proben wurden eigene Prüfkörper gleicher Höhe verwendet, um der Setzung der Versatzstoffe entgegenzuwirken bzw. gleiche Verhältnisse zu schaffen. Des Weiteren wurden von einer der Probemischungen auch Zylinderproben mit den Maßen D $\times \mathrm{H}=150 \times 300 \mathrm{~mm}$ erstellt und geprüft, um zu erfahren, ob eine weitere Probenvergrößerung andere oder präzisere Ergebnisse liefert.

Nach der Herstellung der Proben wurden diese bis zu ihrer Prüfung untertägig gelagert. Diese Lagerungsart ist gewählt worden, da sie eine konstante Temperatur und Luftfeuchtigkeit bietet, welche für die Vergleichbarkeit der Prüfergebnisse notwendig sind. Wie bereits in einigen Studien 
über Versatz beschrieben wurde, haben die Lagerungsart und Lagerungsdauer große Auswirkungen auf die erzielte Versatzfestigkeit [4]. So konnte beispielsweise bei Versuchen mit bindemittelhaltigem Spülversatz bei zwischen $10^{\circ} \mathrm{C}$ und $30^{\circ} \mathrm{C}$ gelagerten Proben kein wesentlicher Unterschied in der 28-Tage-Festigkeit festgestellt werden. Jedoch erreichten Proben, die bei über $40^{\circ} \mathrm{C}$ gelagert wurden, die 3-Monatsfestigkeit bereits nach 28 Tagen [6]. Unter der Annahme, dass sich bindemittelhaltiger Pumpversatz ähnlich verhält, ist dies eine wichtige Erkenntnis, da im geschilderten Betrieb die Temperatur während der Versatzkampagnen durch die Hydrationswärme deutlich ansteigt. Dies weist erneut auf die Tatsache hin, dass die Ergebnisse aus Laborversuchen nur bedingt auf die Anwendung im untertägigen Bergbau zutreffen und nur als Richtwert herangezogen werden können.

\section{Prüfablauf}

Für die Überprüfung der Festigkeitsentwicklung wurden jeweils 5 Proben jeder Mischung 14, 21, 28, 56 und 90 Tage nach der Probenerzeugung auf ihre UCS geprüft. Die Zylinderproben mit einem Durchmesser von $115 \mathrm{~mm}$ wurden hierbei mit der firmeneigenen Druckpresse geprüft. Die Zylinderproben mit einem Durchmesser von $150 \mathrm{~mm}$ wurden extern geprüft, da mit der firmeninternen Prüfpresse nur Proben mit einer Höhe von 240 mm geprüft werden konnten. Hier ist anzumerken, dass bei der betriebsinternen Prüfpresse die Belastungsgeschwindigkeit der Probe während der Prüfung händisch reguliert werden musste. Sie konnte nicht wie bei größeren Pressen voreingestellt werden, was Einfluss auf die Genauigkeit der Prüfergebnisse haben kann.

\subsection{Auswertung der Ergebnisse}

Bei der Druckfestigkeitsprüfung der vorliegenden Versatzmischungen hat sich herausgestellt, dass die Festigkeitsentwicklung in den ersten 90 bzw. 120 Tagen in zwei Bereiche eingeteilt werden kann (Abb. 1).
Bei allen drei Mischungen hat sich in den ersten 14 Tagen ein steiler Anstieg in der Festigkeit gezeigt, der dann bis zum Tag 28 abflachte. Ab der vierten Woche weisen alle Mischungen einen fast linearen Anstieg in der Festigkeit auf. Dieser konnte bei der "Mischung 1 " aufgrund von überzähligen Proben bis zum 120. Tag nach der Probenahme verfolgt werden, wie in Abb. 1 dargestellt ist.

Anhand des Diagramms in Abb. 1 ist deutlich zu erkennen, dass durch Erhöhung der Menge an verwendetem Zement die Festigkeitsbildung in den ersten 14 Tagen beschleunigt wird. Ab der vierten Woche ist der Unterschied im Festigkeitsanstieg der einzelnen Mischungen gering. In dieser Arbeit wurde der Festigkeitsverlauf zwar nur in den ersten 90 bzw. 120 Tagen überprüft, jedoch haben Prüfungen aus vergangenen Versatzkampagnen, die zum Teil 280 Tage nach Herstellung des Versatzes erfolgten, gezeigt, dass die Festigkeit weiter zunimmt. Die Annahme einer stetigen Festigkeitszunahme über viele Monate wird somit bekräftigt.

\subsection{Genauigkeit der Prüfergebnisse}

Ein wesentlicher Bestandteil dieser Arbeit war die Optimierung des Versatzprüfverfahrens. Hier hat sich gezeigt, dass durch Vergrößerung der Proben von D x H = $100 \times 200 \mathrm{~mm}$ auf $\mathrm{D} \times \mathrm{H}=115 \times 230 \mathrm{~mm}$ die Festigkeitsschwankungen von $10-20 \%$, bezogen auf den Mittelwert einer Probencharge, auf 4 bis $8 \%$ reduziert werden konnten. Bei der Prüfung von zylindrischen Proben mit den Maßen D x H = $150 \times 300$ mm waren die relativen Standardabweichungen zwar geringer als die der intern geprüften Zylinder, jedoch haben die Proben an den Prüftagen im Mittel gleich hohe Festigkeiten erreicht.

\section{Schlussfolgerung}

Durch Erhöhung des Probendurchmessers bei den Zylinderformen von $100 \mathrm{~mm}$ auf $115 \mathrm{~mm}$ und durch die Anfertigung von gleich großen Probekörpern, aus denen jeweils nur eine Probe erstellt wurde, konnten bei der einaxialen Druckfestigkeitspüfung Festigkeitswerte erzielt werden,
Abb. 1: Einaxiale Druckfestigkeit in Abhängigkeit der Lagerungsdauer und der Bindemittelzusammensetzung der Mischung

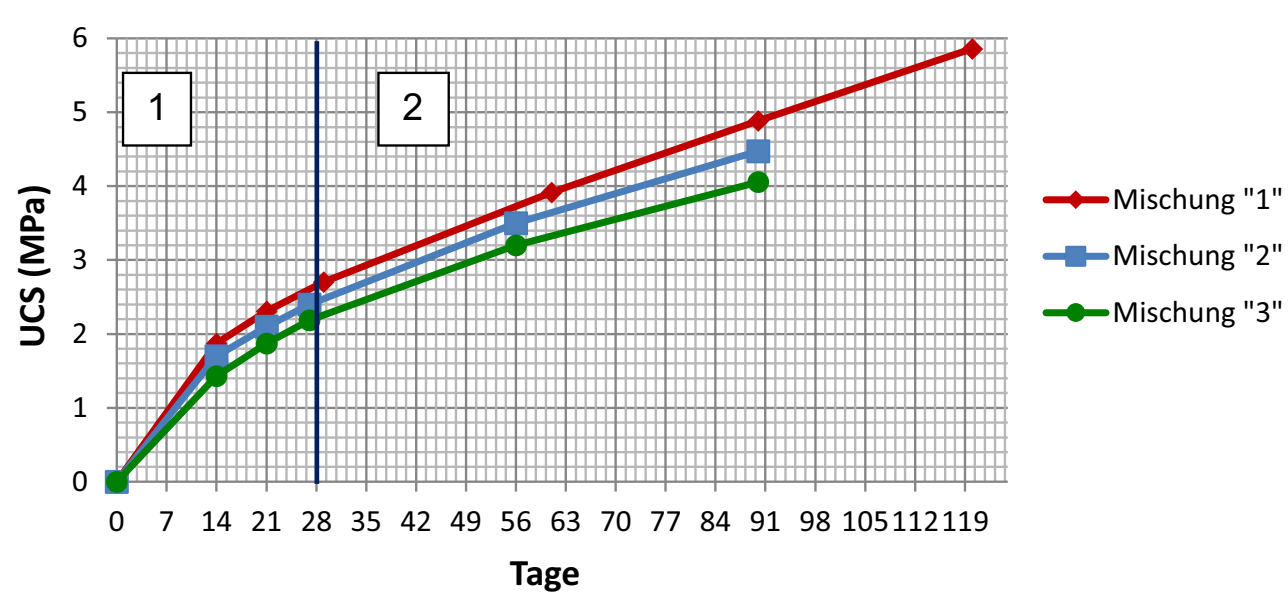


die mit einer relativen Standardabweichung von maximal $7-8 \%$ je Mischung schwanken. Dies ist zwar eine relativ hohe Schwankung, jedoch haben die erzielten Festigkeitswerte aus bisherigen Versatzkampagnen mit $10 \%$ bis $20 \%$ relativer Standardabweichung eine deutlich höhere Schwankung ergeben. Durch externes Prüfen von Zylinderproben mit den Maßen $150 \times 300 \mathrm{~mm}$ konnte gezeigt werden, dass durch Prüfpressen, bei denen die Belastungsgeschwindigkeit genau voreingestellt werden kann und durch die größeren Proben die Festigkeitsschwankung mit 3,5\%, bezogen auf den Mittelwert, reduziert werden konnte. Im Vergleich mit den erzielten Festigkeitswerten aus der internen Prüfung sieht man jedoch, dass eine Erhöhung der Probengröße keine Veränderung in den Ergebnissen der einaxialen Druckfestigkeit bewirkt, sondern nur die Genauigkeit der Ergebnisse erhöht.

Zusammengefasst kann gesagt werden, dass die in dieser Arbeit beschriebene Erstellung und Lagerung von Zylinderproben mit einer Größe von D x H $=114 \times 230 \mathrm{~mm}$ eine ausreichend genaue Prüfung für bindemittelhaltigen Pumpversatz im betreffenden Bergbau darstellt. Es ist jedoch hervorzuheben, dass diese Ergebnisse, die bei Untersuchungen im Labor festgestellt wurden, von den tatsächlichen Festigkeiten des Versatzkörpers im AbbauhohIraum deutlich abweichen können, wie in Kap. 2 beschrieben wurde.

Durch das in dieser Arbeit durchgeführte Probenprogramm konnte gezeigt werden, wie die Festigkeitsentwicklung von drei unterschiedlichen Mischungen in den ersten 90 bzw. 120 Tagen nach der Probenherstellung verläuft. Hierbei ist festzuhalten, dass gerade in den ersten 4 Wochen nach der Versatzeinbringung die verwendete Zementmenge eine wichtige Rolle in der Festigkeitsentwicklung spielt. Wird der Versatzkörper in den ersten 56 bis 90 Tagen freigestellt, zum Beispiel durch Abbau des unmittelbar an den Versatzkörper grenzenden Gebirgsmaterials, so kann die dafür erforderliche Festigkeit nur durch Erhöhung der Zementmenge erzielt werden. Die tatsächlich erreichte „Endfestigkeit" des Versatzkörpers einer bestimmten Mischung kann anhand der Entwicklung in den ersten 90 Tagen nicht präzise vorhergesagt werden, da die Festigkeitszunahme zu diesem Zeitpunkt noch nicht zur Gänze abgeschlossen ist.

Open access funding provided by Montanuniversität Leoben

Open Access Dieser Artikel wird unter der Creative Commons Namensnennung 4.0 International Lizenz (http://creativecommons.org/licenses/ by/4.0/deed.de) veröffentlicht, welche die Nutzung, Vervielfältigung, Bearbeitung, Verbreitung und Wiedergabe in jeglichem Medium und Format erlaubt, sofern Sie den/die ursprünglichen Autor(en) und die Quelle ordnungsgemäß nennen, einen Link zur Creative Commons Lizenz beifügen und angeben, ob Änderungen vorgenommen wurden.

\section{Literatur}

1. Hohl, W.; Frömmer, T.: Die Versatzrichtlinie des BVÖ: Ein Leitfaden für die zeitgemäße Planung eines Versatzsystems, BHM Berg- und Hüttenmännische Monatshefte, 158 (2013), S. 410-423

2. Moser, A.: State of the Art of Backfill Technology in Underground Mining Excavations. Diplomarbeit, Montanuniversität Leoben, 2015

3. Moser, A.: Stand der Technik der Versatztechnologie im untertägigen Bergbau, BHM Berg- und Hüttenmännische Monatshefte, 161 (2016), S. 345-350

4. Hohl, W. (2009): Stand der Prüf- und Untersuchungsverfahren für Versatz, BHM Berg- und Hüttenmännische Monatshefte, 154 (2009), S. 74-79

5. Brady, B. H. G.; Brown, E. T.: Rock Mechanics for underground mining, $3^{\text {rd }}$ edition, Springer Science, 2005

6. Helms, W.: Eigenschaften, Anwendung und gebirgsmechanische Auswirkungen des bindemittelverfestigten Versatzes, Habil.-Schrift, Clausthal, TU Bergakademie, 1988 